\title{
Minireview
}

\section{Targeted radiotherapy of brain tumours}

\author{
MR Zalutsky*, I \\ 'Department of Radiology, Duke University Medical Center, PO Box 3808, Durham, NC 277I 0, USA
}

The utility of external beam radiotherapy for the treatment of malignant brain tumours is compromised by the need to avoid excessive radiation damage to normal CNS tissues. This review describes the current status of targeted radiotherapy, an alternative strategy for brain tumour treatment that offers the exciting prospect of increasing the specificity of tumour cell irradiation. British Journal of Cancer (2004) 90, |469- |473. doi: I0.1038/sj.bjc.660 I77I www.bjcancer.com

Published online 6 April 2004

(C) 2004 Cancer Research UK

Keywords: glioblastoma multiforme; radiotherapy; radioimmunotherapy; glioma; anaplastic astrocytoma

Even with aggressive multi-modality treatment strategies, the life expectancy for patients with glioblastoma multiforme (GBM), the most common and virulent primary brain tumour, is less than a year from the time of diagnosis (Stewart, 2002). The vast majority of glioma patients experience local recurrence, with a median survival of only 16-24 weeks for those with recurrent disease (Wong et al, 1999). Conventional radiotherapy continues to play a primary role in brain cancer treatment; however, its lack of tumour specificity is a severe limitation of this form of therapy. Owing to its nonspecific nature, toxicity to normal brain limits the radiation dose that can be delivered to tumour cells, and compromises the quality of life of the few longer-term survivors.

An emerging approach for brain tumour treatment is targeted radiotherapy, a strategy that utilises a molecular vehicle to selectively deliver a radionuclide to malignant cell populations. An important consideration is to match the decay properties of the radionuclide with the characteristics of the tumour. The two most commonly utilised radionuclides in targeted radiotherapy, ${ }^{131} \mathrm{I}$ and ${ }^{90} \mathrm{Y}$, emit beta particles, which deposit $95 \%$ of their energy within 0.992 and $5.94 \mathrm{~mm}$, respectively (Hopkins et al, 1998). Radiation of this type might be best suited for treating tumour that may be present in the $2-\mathrm{cm}$ rim around the brain tumour resection cavity, where most gliomas recur. On the other hand, alpha particles have a tissue range of only a few cell diameters and might be ideal for elimination of the small clusters or single glioma cells that can occur $4-7 \mathrm{~cm}$ from the primary tumour site (Goldbrunner et al, 1999). Alpha particles could potentially enhance the therapeutic potential for minimal residual disease by maximising the radiation dose received by these relatively small tumour foci, while sparing normal CNS tissues.

Although many types of labelled molecules have been explored for targeted cancer radiotherapy, investigations in brain tumour patients have almost exclusively utilised monoclonal antibodies (mAbs) as the targeting vehicle. Brain-tumour-associated molecular targets that have been evaluated for radioimmunotherapy include the epidermal growth factor receptor (Brady et al, 1992) and the human neural cell adhesion molecule (NCAM), which is

*Correspondence: MR Zalutsky; E-mail: zalut00 I@mc.duke.edu Received 16 October 2003; revised 4 February 2004; accepted 16 February 2004; published online 6 April 2004 present both on glioma as well as normal neural tissue (Hopkins et al, 1998). However, the vast majority of targeted radiotherapy studies in brain tumour patients have utilised radiolabelled mAbs reactive with the tenascin molecule (Table 1 ).

\section{TENASCIN AND ANTI-TENASCIN MABS}

Tenascin- $C$ is a hexabrachion polymorphic glycoprotein that is overexpressed in the extracellular matrix in high-grade gliomas as well as other tumour types. The expression of tenascin increases with advancing tumour grade, with more than $90 \%$ of glioblastoma multiforme biopsies exhibiting very high levels of tenascin expression. Tenascin-C expression occurs primarily around tumour-supplying blood vessels, with this staining pattern becoming more pronounced with increasing tumour grade (Herold-Mende et al, 2002). Furthermore, in WHO II and III gliomas, there appears to be a correlation between perivascular staining and earlier tumour recurrence. The $\mathrm{mAb} \mathrm{BC}-4$ binds to an epitope within the epidermal growth factor (EGF)-like repeat found on all tenascin isoforms, while BC-2 reacts with an epitope found on the alternatively spliced fibronectin type III repeats A1 and A4, which share $83 \%$ homology (Balza et al, 1993). The antitenascin mAb developed by our group, 81C6 (Bourdon et al, 1983), binds to an epitope within the alternatively spliced fibronectin type III CD region. In the following sections, the current status of targeted brain tumour radiotherapy with these mAbs, both directly labelled and as part of a pretargeting strategy, will be reviewed.

\section{ANTI-TENASCIN MAB 81C6}

In order to exploit the potential utility of tenascin as a molecular target for radioimmunotherapy, mAb 81C6, a murine $\operatorname{IgG}_{2 b}$, was developed. The ability of murine 81C6 to selectively localise and treat human glioma xenografts was investigated extensively in rodent models before the initiation of clinical studies with this labelled mAb (Wikstrand et al, 2001). Three observations from diagnostic-level investigations performed in glioma patients had an influence on the design of our subsequent targeted radiotherapy protocols. First, levels of ${ }^{131}$ I-labelled $81 \mathrm{C} 6$ in tumour biopsies were up to five times higher than a co-injected ${ }^{125}$ I-labelled nonspecific $\mathrm{mAb}$, and up to 200 times higher than those in normal 
Table I Summary of targeted radiotherapy clinical trials in brain tumour patients

\begin{tabular}{|c|c|c|c|c|c|}
\hline Radiotherapeutic & Design & Patients $^{\mathbf{a}}$ & Toxicities $^{\mathbf{b}}$ & Survival (weeks) & Reference \\
\hline$|-| 3|8| C 6$ murine $m A b$ & Phase I & 34 (26 GBM) recurrent & Neuro Heme & 60 (56 GBM) & Bigner et al (1998) \\
\hline$|-| 3|8| C 6$ murine $m A b$ & Phase I & 42 (32 GBM) newly diagnosed & Neuro & 79 (69 GBM) & Cokgor et al (2000) \\
\hline$|-| 3|8| C 6$ murine mAb & Phase II & 33 (27 GBM) newly diagnosed & Neuro Heme & 87 (79 GBM) & Reardon et al (2002) \\
\hline At-2II $81 \mathrm{C} 6$ chimeric mAb & Phase I & 17 (I4 GBM) recurrent & None & 60 & Zalutsky et al (2002) \\
\hline$|-| 3 \mid \mathrm{BC}-2$ or $\mathrm{BC}-4 \mathrm{mAb}$ & Phase II & 91 (74 GBM) & None & $>184 \mathrm{AA}$ & Riva et al (2000) \\
\hline $3-10$ cycles & & $\begin{array}{l}44 \text { recurrent } \\
47 \text { newly diagnosed }\end{array}$ & & 76 GBM & \\
\hline $\begin{array}{l}\text { Y-90 BC- } 2 \text { or BC- } 4 \text { mAb } \\
3-5 \text { cycles }\end{array}$ & Phase II & $43(35 \mathrm{GBM})$ & None & $\begin{array}{l}360 \text { AA } \\
80 \text { GBM }\end{array}$ & Riva et al (2000) \\
\hline $\begin{array}{l}\text { Y-90 or I- I3I BC-4 mAb } \\
\text { Mean, three cycles }\end{array}$ & Phase II & 37 (24 GBM) & Neuro & 68 GBM & Goetz et al (2003) \\
\hline $\begin{array}{l}\text { BC-4 mAb, Y-90 biotin } \\
\text { two cycles }\end{array}$ & Phase I & 24 (I6 GBM) recurrent & Neuro & $\begin{array}{l}76 \mathrm{AA} \\
50 \mathrm{GBM}\end{array}$ & Paganelli et al (200I) \\
\hline $\begin{array}{l}\text { BC-4 mAb, Y-90 biotin } \\
\text { two cycles }\end{array}$ & Phase II & 8 GBM & None & 134 & Grana et al (2002) \\
\hline
\end{tabular}

${ }^{a} \mathrm{GBM}=$ glioblastoma multiforme; $\mathrm{AA}=$ anaplastic astrocytoma. ${ }^{\mathrm{b}} \mathrm{Neuro}=$ neurological toxicity; Heme = haemtatological toxicity.

brain (Zalutsky et al, 1989). Thus, within the brain, uptake of ${ }^{131} \mathrm{I}$ labelled 81C6 was both selective and specific. Second, intracarotid injection did not significantly increase tumour delivery compared with intravenous administration (Zalutsky et al, 1990). And third, an $\mathrm{mAb}$ protein escalation study demonstrated that intravenous administration could not be used to deliver therapeutically useful levels of labeled $\mathrm{mAb}$ to tumour without excessive dose to liver and spleen, normal organs that express tenascin (Schold et al, 1993). This led us to focus on a compartmental approach involving direct administration of radiolabelled 81C6 mAb into surgically created tumour resection cavities.

\section{Iodine-131-labelled 81C6 clinical trials}

Our clinical experience with ${ }^{131}$ I-labelled $81 \mathrm{C} 6 \mathrm{mAb}$ currently includes over 300 patients who have received the labelled mAb by direct injection into a surgically created resection cavity via a Rickham reservoir and catheter placed at the time of resection. The entry criteria for parallel phase I studies in patients with recurrent and newly diagnosed malignant glioma included: histopathological confirmation of diagnosis, demonstration of tumour reactivity with $81 \mathrm{C} 6$ by immunohistochemistry, a maximum of $1 \mathrm{~cm}$ residual enhancement on postoperative MRI, and tumor localisation within the supratentorial compartment. Patency of the catheter and intactness of the resection cavity was confirmed by radionuclide imaging prior to treatment, and patients received a saturated solution of potassium iodide to block thyroid uptake of radioiodine that might be released from the labelled mAb. Most patients received systemic chemotherapy after the radioimmunotherapy procedure, and newly diagnosed patients also received external beam radiotherapy.

The phase I study of recurrent disease enrolled 34 patients, including 26 with GBM (Bigner et al, 1998). The administered dose of ${ }^{131}$ I-labelled $81 \mathrm{C} 6$ ranged from 740 to $4440 \mathrm{MBq}(20-120 \mathrm{mCi})$, and a maximum tolerated dose of $3700 \mathrm{MBq}(100 \mathrm{mCi})$ was established. Dose-limiting toxicity was neurologic. The median survival for patients with recurrent GBM and for all patients treated was 56 and 60 weeks, respectively. In the parallel phase I study in patients with newly diagnosed brain tumours, a total of 42 patients were treated including 32 with glioblastoma multiforme (Cokgor et al, 2000). The administered activity of ${ }^{131}$ I-labelled $81 \mathrm{C} 6$ ranged from 740 to $6660 \mathrm{MBq}(20-180 \mathrm{mCi})$. A maximum tolerated dose of $4440 \mathrm{MBq}(120 \mathrm{mCi})$ was established, with doselimiting toxicity again being neurologic. Reversible haematologic toxicity was observed in seven patients. Patient-specific dosimetry calculations indicated that the $2-\mathrm{cm}$ thick region surrounding the resection cavity interface received an average radiation dose of 32
Gy (range 2-59 Gy) (Akabani et al, 2000). The median survival for patients with GBM and all patients was highly encouraging at 69 and 79 weeks, respectively.

A phase II trial was then conducted in patients with newly diagnosed malignant glioma at an administered activity of $4440 \mathrm{MBq}(120 \mathrm{mCi})$ of ${ }^{131} \mathrm{I}$-labelled murine 81C6 (Reardon et al, 2002). In all, 33 patients were enrolled including 27 with GBM. Treatment-related neurotoxicity was observed in five patients and reversible haematologic toxicity occurred in nine patients. It should be noted that irreversible neurotoxicity was associated with the resection cavity being either contiguous or adjacent to the compromised CNS functional centre. The median survival achieved for patients with GBM and all patients enrolled in this trial was 79 and 87 weeks, respectively. These results were compared to those predicted by a recursive partitioning model (Curran et al, 1993). For example, this model predicted a median survival of 55 weeks for newly diagnosed GBM patients less than 50 years old, while in our study an 87-week median survival was achieved. Likewise, newly diagnosed GBM patients over 50 years old with a Karnofsky performance status greater than $70 \%$ were predicted to have a median survival of 39 weeks compared with 65 weeks for those in that category in our study.

Other methodologies such as stereotactic radiosurgery and ${ }^{125} \mathrm{I}$ interstitial brachytherapy also have been investigated for delivering a boost radiation dose to tumour, and the prolonged survival achieved in this phase II study compared favourably to these approaches. However, an important distinction between targeted radiotherapy with ${ }^{131}$ I-labelled $81 \mathrm{C} 6$ and these other techniques was with regard to the need for re-operation to debulk radiation necrosis and to relieve symptomatic mass effect. Re-operation rates for brachytherapy and radiosurgery have been reported to be in the $30-60 \%$ range, while only $2 \%$ of the 109 combined patients on our phase I and II trials required re-operation for symptomatic radionecrosis.

Owing to regulatory constraints, radioiodinated $\mathrm{mAb}$ was given based on ${ }^{131}$ I activity rather than on a calculated radiation dose to the resection cavity margins. Dosimetry calculations indicated that this critical parameter could vary considerably due to differences in the rate of clearance of radioactivity from the resection cavity and the resection cavity volume. In the 42 patients entered on the phase I newly diagnosed trial, radionuclide residence times in the cavity ranged from 10 to $113 \mathrm{~h}$, and cavity volumes from 2 to $81 \mathrm{~cm}^{3}$ (Akabani et al, 2000). This led to average radiation dose deposited in the $2-\mathrm{cm}$ resection cavity margin ranging from 3 to 59 Gy.

A 16-patient subset of these newly diagnosed glioma patients experienced progressive changes on serial MRI images and had 
biopsies, permitting an investigation of the relationship between histopathology and the radiation dose delivered to the $2-\mathrm{cm}$ cavity margin. Patients receiving a dose less than $44 \mathrm{~Gy}$ generally had tumour recurrence, while those receiving more than $44 \mathrm{~Gy}$ to the 2$\mathrm{cm}$ margin had a greater incidence of radiation necrosis. In an attempt to maximise local tumour control while minimising normal brain radionecrosis, we have now initiated another phase I study in which newly diagnosed patients are given an activity level of ${ }^{131}$ I-labelled $81 \mathrm{C} 6$, based on a prior dosimetry study, calculated to deliver an average of $44 \mathrm{~Gy}$ to the resection cavity margins. To date, 17 patients have been treated on this protocol; however, the median follow up period is not sufficient to provide meaningful survival data.

\section{Astatine-211 labelled chimeric $81 \mathrm{C6}$ clinical trial}

An important variable for targeted brain tumour radiotherapy is the nature of the radiation emitted by the radionuclide. Although use of molecules labelled with ${ }^{131} \mathrm{I}$ or other beta emitters can increase the selectivity of tumour cell irradiation compared with external beam irradiation, both approaches have similar radiobiological effectiveness. An advantage of alpha particles such as those emitted by the 7.2-h half-live radiohalogen 211 At is that they are high linear energy transfer (LET) radiation, with considerably higher cytotoxicity. Cell culture experiments have demonstrated that human tumour cell lines could be killed with only a few alpha particle traversals per cell (Zalutsky and Vaidyanathan, 2000). Furthermore, the cytotoxicity of alpha particles is nearly independent of dose rate, oxygen concentration and cell cycle stage. As tenascin expression is perivascular, combining anti-tenascin mAb 81C6 with a radionuclide that emits short-range radiation might be a particularly effective approach, in that it could act as a vascular targeted therapeutic (Akabani et al, 2002). In this way, it might be possible to also kill tumour cells indirectly by compromising their blood supply.

A phase I trial is currently being performed to determine the maximum tolerated dose, pharmacokinetics and objective responses to ${ }^{211} \mathrm{At}$-labelled chimeric $81 \mathrm{C} 6$ administered into surgically created glioma resection cavities in recurrent glioma patients (Zalutsky et al, 2002). The chimeric construct has human IgG2 constant region domains and was selected for this protocol because its stability in vivo was shown to be considerably higher than that of murine 81C6 (Reist et al, 1998). The ${ }^{211}$ At was produced at the Duke University Medical Center cyclotron and the mAb was labelled with preservation of immunoreactivity by reaction with $N$-succinimidyl 3-[ $\left.{ }^{211} \mathrm{At}\right]$ astatobenzoate. To date, 17 patients (three anaplastic oligodendroglioma, 14 glioblastoma multiforme) have received $10 \mathrm{mg}$ of $\mathrm{mAb}$ labelled with escalating activities ( $74 \mathrm{MBq}, n=5 ; 148 \mathrm{MBq}, n=6 ; 248 \mathrm{MBq}$, $n=5 ; 370 \mathrm{MBq}, n=1)$ of ${ }^{211}$ At-labelled chimeric $81 \mathrm{C} 6$. As this was the first clinical trial of any ${ }^{211}$ At-labelled radiotherapeutic, demonstration of in vivo stability and safety were particularly important. Serial blood counting and imaging were performed and indicated very low levels of leakage of ${ }^{219}$ At from the surgical resection cavity. Less than $0.2 \%$ of the injected dose was found in the blood pool and more than $95 \%$ of the ${ }^{211} \mathrm{At}$ decays occurred within the tumour resection cavity. Cavity interface radiation doses were in the range of $150-35000 \mathrm{~Gy}$ ( $2986 \mathrm{~Gy}$ average dose) compared with $0.01 \mathrm{~Gy}$ for normal organs including tenascin-expressing spleen and liver. Encouraging responses have been obtained with a median survival of 60 weeks observed in all patients. Particularly encouraging is the fact that two patients with recurrent GBM survived for more than 150 weeks, and a patient with recurrent anaplastic oligodendroglioma is now approximately 215 weeks from treatment. The maximum tolerated dose of ${ }^{21}$ At-labelled chimeric $81 \mathrm{C} 6$ has yet to be defined.

\section{STUDIES WITH BC-2 AND BC-4 ANTI-TENASCIN MABS}

(Riva et al, 2000) have been evaluating the efficacy of ${ }^{131}$ I-labelled and ${ }^{90}$ Y-labelled $\mathrm{BC}-2$ and $\mathrm{BC}-4 \mathrm{mAbs}$ for the locoregional treatment of malignant gliomas. In these protocols, no distinction was made between the two mAbs. The phase II study with ${ }^{131} \mathrm{I}$ involved 91 patients including 74 with glioblastoma and nine with anaplastic astrocytoma. At the time of treatment, 52 patients were classified as having small (less than $2 \mathrm{~cm}^{3}$ ) or undetectable residual tumour, with the remainder having a larger tumour mass. The study population consisted of 47 newly diagnosed and 44 recurrent tumours. Patients received three to 10 cycles of ${ }^{131} \mathrm{I}$-labelled mAb, at intervals of either 1 or 3 months, with a cumulative administered activity of up to $20.35 \mathrm{GBq}(550 \mathrm{mCi})$. The median survival was $>46$ months in anaplastic astrocytoma and 19 months in glioblastoma, with no distinction made between newly diagnosed and recurrent patients groups. The response rate in glioblastoma patients was better in those with small volume (56.7\%) compared with larger tumours (17.8\%).

A subsequent study was performed using ${ }^{90} \mathrm{Y}$ in order to investigate the potential effects of using a radionuclide emitting beta particles with greater tissue penetration (Riva et al, 2000). In this phase II investigation, 43 patients were treated, including six with anaplastic astrocytoma and 35 with glioblastoma. In all, 16 were classified as having small volume or minimal disease at the time of treatment. Patients received between three and five cycles of ${ }^{90} \mathrm{Y}$-labelled mAbs with a cumulative activity of up to $3.145 \mathrm{GBq}$ $(85 \mathrm{mCi})$. The median survival for patients with anaplastic astrocytoma and glioblastoma was 90 months and 20 months, respectively. The response rate in glioblastoma patients was $26.3 \%$ in those with bulky disease compared with $56.3 \%$ for those with smaller lesions.

In a more recent study, the therapeutic potential of ${ }^{131} \mathrm{I}-$ and ${ }^{90} \mathrm{Y}-$ labelled BC-4 mAb were evaluated in 37 patients, consisting of 13 with astrocytoma WHO grade III and 24 with WHO grade IV histology (Goetz et al, 2003). Multiple cycles of labelled mAbs were administered (mean, three per patient) at various activity levels. The median survival for glioblastoma patients was 17 months. No attempt was made to stratify analyses according to the radionuclide used or whether the patients had recurrent or newly diagnosed lesions.

These clinical studies are important in that they confirm the potential of locoregionally administered labelled mAbs as a means for improving the survival of patients with malignant brain tumours. The low incidence of side effects, even after multiple cycles, also is encouraging. However, it remains to be ascertained whether use of the higher energy beta emitter ${ }^{90} \mathrm{Y}$ and multiple cycles of labelled $\mathrm{mAb}$ results in a significant improvement in therapeutic efficacy compared with a single dose of ${ }^{131}$ I-labelled $\mathrm{mAb}$.

\section{PRETARGETED RADIOIMMUNOTHERAPY}

One of the limitations of directly labelled mAbs for targeted radiotherapy is that as a consequence of their macromolecular size they diffuse slowly through tissue, hampering their delivery to tumour cells distant from their site of injection. An attractive strategy to compensate for the large size of mAbs is pretargeting, a procedure in which the $\mathrm{mAb}$ is administered first, followed after an appropriate time interval by the injection of a radiolabelled low molecular weight vehicle. The most common approach attempts to exploit the extraordinarily high affinity of avidin or streptavidin for the $244 \mathrm{Da}$ vitamin, biotin. A three-step avidin-biotin based regimen has been investigated in glioma patients who first received biotinylated BC-4 mAb, followed $24 \mathrm{~h}$ later by avidin, and finally, after an additional $18 \mathrm{~h}$, a ${ }^{90} \mathrm{Y}$-labelled biotin conjugate. 
The first trial with this pretargeting approach was performed in patients with recurrent glioma and the three reagents were administered via a catheter placed into the surgical resection cavity (Paganelli et al, 2001). In all, 16 patients with glioblastoma and eight with anaplastic astrocytoma were treated with two cycles administered 8-10 weeks apart. The maximum tolerated dose was $1.11 \mathrm{GBq}(30 \mathrm{mCi})$ of ${ }^{90} \mathrm{Y}$-labelled DOTA, biotin with neurologic toxicity being the dose-limiting factor. After the second operation, median survival was 19 and 11.5 months in patients with anaplastic astrocytoma and glioblastoma, respectively.

Recently, the efficacy of this pretargeting radioimmunotherapy protocol was evaluated in an adjuvant setting (Grana et al, 2002). Newly diagnosed patients, 17 with grade III glioma and 20 with glioblastoma, received surgery and external beam radiation. Then, 19 patients received the three reagents in the sequence described above with the ${ }^{90} \mathrm{Y}$-labelled biotin being given at a dose of $2.2 \mathrm{GBq} \mathrm{m}^{-2}$, with the remaining 18 patients serving as controls. Unlike the initial clinical study, all reagents were administered via the intravenous route instead of directly into the surgical resection cavity. The median survival estimated for the grade IV glioma patients was 8 months in the control group $(n=12)$ and 33.5 months in the treated group $(n=8)$. Only two of the 11 treated grade III patients had died at the time of that publication.

The results obtained with this pretargeting protocol are highly encouraging, particularly in light of the fact that significant survival prolongation could be obtained even when then labelled compound was administered intravenously. Furthermore, it was discovered after completion of this trial that the $\mathrm{BC}-4 \mathrm{mAb}$ hybridoma clone produced an additional nonfunctional light chain (De Santis et al, 2003). To solve this problem, a new anti-tenascin $\mathrm{mAb}, \mathrm{ST} 2146$, has been generated. A multi-centre clinical trial is currently being planned to evaluate the therapeutic potential of pretargeted radioimmunotherapy, with ST2146 mAb providing the tenascin targeting component.

\section{RADIOLABELLED PEPTIDES}

Owing to the infiltrative nature of glioma, methods must be devised for improving the delivery of targeted radiotherapy to tumour cells that are distant from the primary lesion. One of the limitations of mAbs is that they diffuse slowly through tissue as a consequence of their large molecular size. Peptides can have molecular weights two orders of magnitude less than intact mAbs and thus are attractive carrier molecules for the targeted irradiation of distant tumour cells. This strategy is currently being investigated in patients with low-grade gliomas, many of which overexpress somatostatin type 2 receptors (Hofer et al, 2001; Schumacher et al, 2002).

\section{REFERENCES}

Akabani G, Cokgor I, Coleman RE, González Trotter D, Wong T, Friedman HS, Garcia-Turner A, Herndon II JE, DeLong D, McLendon RE, Zhao X-G, Pegram CN, Bigner DD, Zalutsky MR (2000) Dosimetry and dose-response relationships in newly diagnosed patients treated with iodine-131-labeled anti-tenacin monoclonal antibody therapy. Int J Radiat Oncol Biol Phys 46: $947-958$

Akabani G, McLendon RE, Bigner DD, Zalutsky M (2002) Vascular targeted endoradiotherapy of tumors using alpha-particle-emitting compounds: theoretical analysis. Int J Radiat Oncol Biol Phys 4: 1259-1275

Balza E, Siri A, Ponassi M, Caocci F, Linnala A, Virtanen I, Zardi L (1993) Production and characterization of monoclonal antibodies specific for different epitopes of human tenascin. FEBS Lett 332: 39-43

Bigner DD, Brown MT, Friedman AH, Coleman RE, Akabani G, Friedman HS, Thorstad WL, Mclendon RE, Bigner SH, Zhao X-G, Pegram CN, Wikstrand CJ, Herndon II JE, Vick NA, Paleologos N, Cokgor I, Provenzale JM, Zalutsky MR (1998) Iodine-131-labeled
Five patients with progressive gliomas (two WHO grade II, three WHO grade III) and five patients with surgically debulked WHO grade II gliomas were treated with the labelled somatostatin analogue $\left[{ }^{90} \mathrm{Y}\right]-\mathrm{DOTA}{ }^{0}-\mathrm{D}-\mathrm{Phe}^{1}-\mathrm{Tyr}^{3}$-octreotide. Patients received between one and five cycles of the labelled peptide at a cumulative activity of $555-7030 \mathrm{MBq}(15-190 \mathrm{mCi})$. Responses of 13-45 months duration were observed in the progressive patients. Disease stabilisation was observed in the five newly diagnosed low-grade glioma patients who received radiolabelled peptide therapy following resection. Side effects included increased seizure frequency and were transient. However, the potential clinical role of target radiotherapy in lower grade gliomas is less clear, because a wait-and-see attitude currently prevails for patients with these malignancies.

\section{CONCLUSIONS AND FUTURE PERSPECTIVES}

In summary, clinical trials, primarily with radiolabelled antitenascin mAbs, have demonstrated the feasibility of performing targeted radiotherapy in glioma patients. By administering the labelled $\mathrm{mAb}$ directly into surgically created resection cavities, a significant survival advantage has been obtained for patients with malignant glioma in comparison with radiation therapy combined with chemotherapy. Furthermore, we have shown that ${ }^{131} \mathrm{I}-$ labelled 81C6 results in significantly lower toxicity than other techniques designed to boost radiation dose to the primary tumour site such as stereotactic radiosurgery or brachytherapy.

It remains to be ascertained whether multiple cycles of targeted radiotherapeutic offer a significant survival advantage compared with single-dose protocols. Likewise, it is not clear whether longer range beta emitters such as ${ }^{90} \mathrm{Y}$ or shorter range beta emitters such as ${ }^{131} \mathrm{I}$ provide the best balance between maximising tumour cell killing and minimising debilitating toxicity to normal brain. The most intriguing challenge for targeted brain tumour radiotherapy will be to develop strategies for delivering curative doses of radiation to small deposits and single glioma cells located centimetres from the primary lesion. Work to date with highly cytotoxic alpha emitters and highly diffusible peptides is encouraging in this regard. Use of micro-infusion techniques such as those being used to treat gliomas with immunotoxins (Nguyen et al, 2003) may also play an important role.

\section{ACKNOWLEDGEMENTS}

Work performed at the author's institution was supported in part by grants from the National Institutes of Health and the Department of Energy. antitenascin monoclonal antibody 81C6 treatment of patients with recurrent malignant gliomas: phase I trial results. J Clin Oncol 16: $2202-2212$

Bourdon MA, Wikstrand CJ, Furthmayr H, Matthews TJ, Bigner DD (1983) Human glioma-mesenchymal extracellular matrix antigen defined by monoclonal antibody. Cancer Res 43: 2796-2805

Brady LW, Miyamoto C, Woo DV, Rackover M, Emrich J, Bender H, Dadparvar S, Steplewski Z, Koprowski H, Black T, Lazzaro B, Nair S, McCormack T, Nieves J, Morabito M, Eshleman J (1992) Malignant astrocytomas treated with iodine- 125 labeled monoclonal antibody 425 against epidermal growth factor receptor: a phase II trial. Int J Radiat Oncol Biol Phys 22: 225-230

Cokgor I, Akabani G, Kuan C-T, Friedman HS, Friedman AH, Coleman RE, McLendon RE, Bigner SH, Zhao X-G, Turner AM, Pegram CN, Wikstrand CJ, Shafman TD, Herndon II JE, Provenzale JM, Zalutsky MR, Bigner DD (2000) Phase I trial results of iodine-131-labeled antitenascin monoclonal 
antibody 81C6 treatment of patients with newly diagnosed malignant gliomas. J Clin Oncol 18: 3862-3872

Curran WJ, Scott CB, Horton J, Nelson JS, Weinstein AS, Fischbach AJ, Chang CH, Rotman M, Asbell SO, Krisch RE, Nelson DF (1993) Recursive partitioning analysis of prognostic factors in three Radiation Therapy Oncology Group malignant glioma trials. J Natl Cancer Inst 85: 704-710 De Santis R, Anastasi AM, Alessio V D, Pellicia A, Albertoni C, Rosi A, Leoni B, Lindstedt R, Petronzelli F, Dani M, Verdoliva A, Ippolito A, Campanile N, Manfredi V, Esposito A, Cassani G, Chinol M, Paganelli G, Carminati P (2003) Novel antitenascin antibody with increased tumour localisation for Pretargeted Antibody-Guided RadiolmmunoTherapy (PAGRIT). Br J Cancer 88: 996-1003

Goetz C, Riva P, Poepperl G, Gildehaus FJ, Hischa A, Tatsch K, Reulen H-J (2003) Locoregional radioimmunotherapy in selected patients with malignant glioma: experiences, side effects and survival times. J NeuroOncol 62: $321-328$

Goldbrunner RH, Bernstein JJ, Tonn J-C (1999) Cell-extracellular matrix interaction in glioma invasion. Acta Neurochir. 141: 295-305

Grana C, Chinol M, Robertson C, Mazzetta C, Bartolomei M, De Cicco C, Fiorenza M, Gatti M, Caliceti P, Paganelli G (2002) Pretargeted adjuvant radioimmunotherapy with Yttrium-90-biotin in malignant glioma patients: a pilot study. Br J Cancer 86: 207-212

Herold-Mende C, Mueller MM, Bonsanto MM, Schmitt HP, Kunze S, Steiner HH (2002) Clinical impact and functional aspects of Tenascin-C expression during glioma progression. Int J Cancer 98: 362-369

Hofer S, Eichhorn K, Freitag P, Reubi JC, Mueller-Brand J, Maecke H, Merlo A (2001) Successful diffusible brachytherapy (dBT) of a progressive lowgrade astrocytoma using the locally injected peptidic vector and somatostatin analogue $\left[{ }^{90} \mathrm{Y}\right]-\mathrm{DOTA}{ }^{0}$-D-Phe ${ }^{1}$-Tyr ${ }^{3}$-octreotide (DOTATOC). Swiss Med Wkly 131: 640-644

Hopkins K, Chandler C, Eatough J, Moss T, Kemshead JT (1998) Directed injection of ${ }^{90} \mathrm{Y}$ moabs into glioma tumor resection cavities leads to limited diffusion of the radioimmunoconjugates into normal brain parenchyma: a model to estimate absorbed radiation dose. Int $J$ Radiat Oncol Biol Phys 40: 835-844

Nguyen TT, Pannu YS, Sung C, Dedrick RL, Walbridge S, Brechbiel MW, Garmestani K, Beitzel M, Yordanov AT, Oldfield EH (2003) Convective distribution of macromolecules in the primate brain demonstrated using computerized tomography and magnetic resonance imaging. J Neurosurg 98: $584-590$

Paganelli G, Bartolomei M, Ferrari M, Cremonesi M, Broggi G, Maira G, Sturiale C, Grana C, Prisco G, Gatti M, Caliceti P, Chinol M (2001) Pretargeted radioimmunotherapy with ${ }^{90} \mathrm{Y}$-biotin in glioma patients: phase I study and preliminary therapeutic results. Cancer Biother Radiopharm 16: $227-235$

Reardon DA, Akabani G, Coleman RE, Friedman AH, Friedman HS, Herndon II JE, Cokgor I, McLendon RE, Pegram CN, Provenzale JM, Quinn JA, Rich JN, Regalado LV, Sampson JH, Shafman TD,
Wikstrand CJ, Wong TZ, Zhao X-G, Zalutsky MR, Bigner DD (2002) Phase II trial of murine ${ }^{131}$ I-labeled antitenascin monoclonal antibody 81C6 administered into surgically created resection cavities of patients with newly diagnosed malignant gliomas. J Clin Oncol 20: $1389-1397$

Reist CJ, Bigner DD, Zalutsky MR (1998) Human IgG2 constant region enhances in vivo stability of anti-tenascin antibody $81 \mathrm{C} 6$ compared with its murine parent. Clin Cancer Res 4: 2495-2502

Riva P, Franceschi G, Riva N, Casi M, Santimaria M, Adamo, M (2000) Role of nuclear medicine in the treatment of malignant gliomas: the locoregional radioimmunotherapy approach. Eur J Nucl Med 27: $601-609$

Schold SC\#\#Jr, Zalutsky MR, Coleman RE, Glantz MJ, Friedman AH, Jaszczak RJ, Bigner SH, Bigner DD (1993) Distribution and dosimetry of I-123-labeled monoclonal antibody 81C6 in patients with anaplastic glioma. Invest Radiol 28: 488-496

Schumacher T, Hofer S, Eichhorn K, Wasner M, Zimmerer S, Freitag P, Probst A, Gratz O, Reubi J-C, Maecke HR, Mueller-Brand J, Merlo A (2002) Local injection of the ${ }^{90} \mathrm{Y}$-labelled peptidic vector DOTATOC to control gliomas of WHO grades II and III: an extended pilot study. Eur J Nucl Med 29: 486-493

Stewart LA (2002) Chemotherapy in adult high-grade glioma: a systematic review and meta- analysis of individual patient data from 12 randomised trials. Lancet 359: $1011-1018$

Wikstrand CJ, Zalutsky MR, Bigner DD (2001) Therapy of brain tumors with radiolabeled antibodies. In Brain Tumor Immunotherapy, Liau LM, Becker DP, Cloughsey TF, Bigner DD (eds) pp 205-229. Totowa, NJ: Humana Press

Wong ET, Hess KR, Gleason MJ, Jaeckle KA, Kyritsis AP, Prados MD, Levin VA, Yung WKA (1999) Outcomes and factors in recurrent glioma patients enrolled onto phase II trials. J Clin Oncol 17: 2572-2578

Zalutsky M, Reardon D, Akabani G, Friedman A, Friedman H, Herndon J, McClendon R, Quinn J, Rich J, Penne K, Sampson J, Shafman T, Wong T, Bigner D (2002) Astatine-211 labeled human/mouse chimeric antitenascin monoclonal antibody via surgically created resection cavities for patients with recurrent glioma: Phase I study. Neuro-Oncol 4: S103 (abstract)

Zalutsky MR, Moseley RP, Coakham HB, Coleman RE, Bigner DD (1989) Pharmacokinetics and tumor localization of 131I-labeled anti-tenascin monoclonal antibody 81C6 in patients with gliomas and other intracranial malignancies. Cancer Res 49: 2807-2813 1989

Zalutsky MR, Moseley RP, Benjamin JC, Colapinto EV, Fuller GN, Coakham $\mathrm{HB}$, Bigner DD (1990) Monoclonal antibody and $\mathrm{F}\left(\mathrm{ab}^{\prime}\right)_{2}$ fragment delivery to tumor in patients with glioma: comparison of intracarotid and intravenous administration. Cancer Res 50: 4105-4110

Zalutsky MR, Vaidyanathan G (2000) Astatine-211-labeled radiotherapeutics: an emerging approach to targeted alpha-particle therapy. Current Pharm Design 6: 1433 - 1455 